\title{
Advanced Diagnostic Methods for High-Fidelity Measurements in Multiphase Flows: State-of-the-Art, Challenges and Outlook
}

\author{
Christos N. Markides \\ Clean Energy Processes (CEP) Laboratory, Department of Chemical Engineering, \\ Imperial College London, U.K \\ c.markides@imperial.ac.uk
}

\section{Extended Abstract}

Multiphase flows are of crucial importance in many and diverse settings and applications across a wide range of scales, including flows in biological systems, atmospheric and marine environments, geological processes, planetary atmospherics, as well as numerous industrial processes, such as in the oil and gas, petrochemical, energy, nuclear, transport, automotive, manufacturing, and food production sectors. In particular, multiphase flows are encountered in important processes such as evaporation, condensation, boiling, mixing, reaction, cavitation, erosion, sedimentation, extraction, and many more.

Experimental techniques based on optical measurement principles have experienced significant growth in recent decades. They are able to provide detailed information with high-spatiotemporal resolution on important scalar (e.g., temperature, concentration, and phase) and vector (e.g., velocity) fields in multiphase flows, as well as interfacial characteristics, which has been instrumental to step-changes in our fundamental understanding of these flows, and the development and validation of advanced models with ever-improving predictive accuracy and reliability. Relevant techniques rely upon optical methods such as direct photography, laser-induced fluorescence, laser Doppler velocimetry/phase Doppler anemometry, particle image/tracking velocimetry, and variants thereof.

This talk will discuss the development of and present results from the application of a range of such experimental methods, including the deployment of simultaneous techniques for the generation of multi-physics, multi-field and multiscale information, discuss the specific challenges faced when attempting to perform such measurements in select multiphase flows, and present a future outlook for these methods that will enable an increasingly complete picture and understanding of relevant flows and associated phenomena, and the design of ever-improving processes, devices and systems. 